\title{
The Human Trochlear and Abducens Nerves at Different Ages - a Morphometric Study
}

\author{
Muthu Ramkumar ${ }^{1}$, Saroj Sharma ${ }^{1}$, Tony G. Jacob ${ }^{1}$, Daya N. Bhardwaj ${ }^{2}$, Tapas C. Nag ${ }^{1}$, Tara Sankar Roy ${ }^{1}$ \\ ${ }^{1}$ Department of Anatomy, All India Institute of Medical Sciences, New Delhi-110608, India \\ ${ }^{2}$ Department of Forensic Medicine, All India Institute of Medical Sciences, New Delhi-110608, India
}

[Received November 14, 2013; Revised February 21, 2014; Accepted March 10, 2014]

\begin{abstract}
The trochlear and abducens nerves (TN and AN) control the movement of the superior oblique and lateral rectus muscles of the eyeball, respectively. Despite their immense clinical and radiological importance no morphometric data was available from a wide spectrum of age groups for comparison with either pathological or other conditions involving these nerves. In the present study, morphometry of the TN and AN was performed on twenty post-mortem samples ranging from 12-90 years of age. The nerve samples were processed for resin embedding and toluidine blue stained thin $(1 \mu \mathrm{m})$ sections were used for estimating the total number of myelinated axons by fractionator and the cross sectional area of the nerve and the axons by point counting methods. We observed that the $\mathrm{TN}$ was covered by a welldefined epineurium and had ill-defined fascicles, whereas the AN had multiple fascicles with scanty epineurium. Both nerves contained myelinated and unmyelinated fibers of various sizes intermingled with each other. Out of the four age groups $(12-20 \mathrm{y}, 21-40 \mathrm{y}, 41-60 \mathrm{y}$ and $>61 \mathrm{y})$ the younger groups revealed isolated bundles of small thinly myelinated axons. The total number of myelinated fibers in the TN and AN at various ages ranged from $1100-3000$ and $1600-7000$, respectively. There was no significant change in the cross-sectional area of the nerves or the axonal area of the myelinated nerves across the age groups. However, myelin thickness increased significantly in the AN with aging (one way ANOVA). The present study provides baseline morphometric data on the human $\mathrm{TN}$ and $\mathrm{AN}$ at various ages.
\end{abstract}

Key words: stereology, morphometry, ocular motor nerves, myelin thickness

Aging can affect vision in many ways. Cataract and presbyopia are the commonest manifestations of aging in the general population. Rarely, diplopia may occur due to various reasons, including abnormalities of motor control of the eyeballs. The oculomotor $(\mathrm{ON})$, trochlear $(\mathrm{TN})$ and abducens (AN) nerves work in tandem to dynamically control the muscles that either fix or move the eyeball in order to attain the optimum view of the world around. Diseases affecting these nerves can cause double-vision, altered visual acuity or a general disorientation in space. These nerves may be involved either individually or collectively in various physiological or pathological conditions that result in defects of gaze and/or of the accommodation reflex. The ON, TN and AN have their motor nuclei in the midbrain and pons that are interconnected by the medial longitudinal fasciculus [1].

On exiting the brainstem, these nerves have a long intracranial course, with AN having the longest in the subarachnoid cisterns, among all cranial nerves [1]. Hence, any disease conditions that affect the skull base and meninges can cause pathological changes (neuropathy) in these nerves. Trauma, especially torsional head injury is a cause for AN injury [2-5] and minor strain can result in isolated IV nerve palsy [6]. Inflammatory and infectious diseases like sarcoidosis, chronic meningitis (tuberculosis or Lyme disease) can cause neuropathy in

*Correspondence should be addressed to: Tara Sankar Roy, Ph.D., Department of Anatomy, All India Institute of Medical Sciences, Ansari Nagar, New Delhi-110608, India. E-mail: tarasankar@hotmail.com, tsroy@aiims.ac.in 
both nerves [6]. The predominance of myelinated fibers in the ocular motor nerves [7] also predisposes the TN and AN to demyelinating diseases like multiple sclerosis [8$10]$.

Vascular aneurysms impinging on these nerves can cause compressive neuropathy [6]. Microvasculopathy secondary to diabetes, atherosclerosis, or hypertension also may cause isolated fourth nerve palsy [11]. Intracranial hypotension - after lumbar puncture has been reported to cause VI nerve palsy [12] . In addition, any space occupying tumour in the cranial cavity can cause compression neuropathy of these nerves. Elevated intracranial pressure causing brainstem displacement can lead to compression of the AN in the Dorello canal and this is responsible for up to $30 \%$ cases of isolated VI nerve palsy observed with pseudotumor cerebri [13]. The TN is also susceptible to trauma because of its long course and delicate structure. The traumatic compression by the overlying tentorium cerebelli is the most frequent cause of acquired TN paralysis [14-16]. Hence, detailed knowledge of each anatomical segment and components of the ocular motor nerves is crucial for investigations, diagnosis and management of various conditions arising in these nerves.

Often, clinical examinations do not reveal the exact site of pathological lesions; therefore various noninvasive techniques such as MRI have been used to study the normal and pathological ocular motor nerves [17]. However, morphometric data derived from noninvasive imaging modalities need to be correlated with actual morphological data in order to be meaningful to diagnosis. Therefore, reliable baseline morphological data that describes size, numbers and distribution of nerve fibers, derived from un-biased stereological methods [18], are required from across the ages to assess changes that may define either diseases or abnormal conditions. To our knowledge and after extensive search of scientific literature we did not find any study that described the TN and $\mathrm{AN}$ at various ages and therefore we have undertaken a detailed morphological study of the connective tissue organization and fiber composition of these nerves using un-biased stereological methods.

\section{MATERIAL AND METHODS}

\section{Sample collection and processing}

Twenty samples each of the pre-cavernous parts of the TN and AN were collected from the mortuary of the department of Forensic Medicine, within 12 hours of death of individuals of both sexes, whose age ranged from the second to the ninth decade without any reported history of diabetes mellitus, neurological diseases or ocular injuries. (Appropriate clearance was obtained from the Ethics Committee at the All India Institute of Medical Sciences, New Delhi, India for this study). The samples were divided into four groups of five nerve samples each according to the available minimum and maximum agesgroup A had nerve samples from 12 to 20; group B, 21 40; group C, 41 - 60 years of age; and group D, 61 years and above.

Initially, the nerve samples were fixed in a solution containing $4 \%$ paraformaldehyde, $1 \%$ glutaraldehyde in $0.1 \mathrm{M}$ phosphate buffer $(\mathrm{pH} 7.4)$ for 48 hours and thereafter they were post-fixed in $1 \%$ osmium tetroxide in $0.1 \mathrm{M}$ phosphate buffer. The samples were processed for resin-embedding (Araldite) and $1 \mu \mathrm{m}$ thick cross-sections of the nerves were obtained using a Reichert Ultramicrotome. The sections were stained with toluidene blue and mounted with araldite resin and were used for morphological observations and stereology. Specimens showing autolysis were not used in the study. The images of the nerves were outlined and traced using a mouse connected to a computer, under the $10 \mathrm{X}$ objective of an Olympus BX61 research microscope attached to CX9000 MBF Biosciences CCD camera which were calibrated and aligned with a calibration grid slide $(250 \mu \mathrm{m}, 25 \mu \mathrm{m})$ provided with the StereoInvestigator software (MicroBrightField Inc., VT, USA). The alignment of the motorized microscope was also calibrated parfocally and parcentrically to permit automatic movement of the image of the tracing when the objective lens is changed.

\section{Morphometry}

\section{Cross-sectional area of the nerve}

The cross sections of the nerves were often of different geometrical shapes and rarely irregular. Automated image analysis systems make the erroneous assumption that all axons are cylindrical tubes, therefore, instead of measuring multiple random diameters of the nerve transverse sections, we chose to measure the area by point counting method [19]. Subsequently, in order to follow the conventional reporting of nerve morphometry we also computed the diameter by the formula ' $d=2 \sqrt{ }(\mathrm{A} / \pi)$ '. The software applied a random point grid within the delineated contour of the nerve. We calculated the area of the cross section by using the formula $\hat{\mathrm{A}}=\mathrm{a} / \mathrm{p} . \mathrm{P}(\hat{\mathrm{A}}=$ area estimated, $\mathrm{a} / \mathrm{p}=$ area per point $(\Delta \mathrm{x} . \Delta \mathrm{y}), \mathrm{P}=$ number of points hitting the cross section) [20]. Since the nerves were embedded in such a manner that the cutting surface was perpendicular to the length of the nerve, most of the crosssections were of uniform dimensions; therefore we repeated the measurements randomly in at least three sections per nerve and the cross-sectional area was expressed as mean $\left(\mu \mathrm{m}^{2}\right)$ (standard deviation-SD). Only 
the transversely sectioned nerve was used for estimation of area.

\section{Designing a random sampling scheme for the fractionator}

In toluidene blue stained sections of the nerves, myelinated fibers appeared as dark blue circles around the space occupied by the axons and only such fibers were counted in this study (Figure 1 and 2). Measurement of unmyelinated fibers has not been attempted because in such sections these fibers cannot be differentiated from connective tissue elements.

We determined the number of myelinated axons in TN and AN by using the fractionator probe of the SteroInvestigator software that uses systematic random sampling method for counting nerve fibers [21-22]. Initially, we undertook a pilot study in order to estimate the counting frame size, the grid size and the number of sections that we would have to analyze systematically in order to get a coefficient of error (CE) below 0.05 [19]. We estimated the CE using the formula CE $(\mathrm{N})=1 / \sqrt{ } \Sigma \mathrm{Q}$ (1-sf), where ' $Q$ ' is the number of profiles counted and ' $\mathrm{sf}$ ' is the sampling fraction. We used a counting frame area 'a' of $625 \mu \mathrm{m}^{2}(25 \mu \mathrm{m} \times 25 \mu \mathrm{m})$ and a sampling grid area (dx.dy) of $62,500 \mu \mathrm{m}^{2}(250 \mu \mathrm{m} \times 250 \mu \mathrm{m})$ for all the nerve samples measured. When we multiply the number of axons counted with the unbiased counting frames ' $Q$ ', by the inverse sampling fraction, $1 / \mathrm{sf}(\mathrm{sf}=\mathrm{a} / \mathrm{dx}$.dy) we get an unbiased estimate of the total number of the myelinated axons ( $\mathrm{N}=\mathrm{Q} .1 / \mathrm{sf})$ [19]. We counted and measured the myelinated axons at a final magnification of $600 \mathrm{X}$ under an oil-immersion objective lens. We used the 'unbiased counting rule' of Gundersen [23] and Sterio [18] that uses two exclusion edges (left and lower) and two inclusion edges (upper and right) on the images while counting [23] (Supplementary Figure 1).

\section{Estimation of axonal area of myelinated fiber and myelin thickness}

We estimated the area of the axons of the myelinated fibers by the $2 \mathrm{D}$ nucleator probe that uses randomly placed isotropic lines intersecting the axonal and myelin profiles (Supplementary Figure 1 inset). The area ' $a$ ' was taken as $\pi l^{2}$, where ' $l$ ' is test line from the centre to the profile boundary [20, 24]. We measured the myelin thickness by subtracting the estimated diameter of the axon from that of the whole nerve fiber.

We divided the fibers of TN and AN into three groups according to their axonal area (small: $<10 \mu \mathrm{m}^{2}$, medium: $10-20 \mu \mathrm{m}^{2}$ and large: $\left.>20 \mu \mathrm{m}^{2}\right)[7]$.

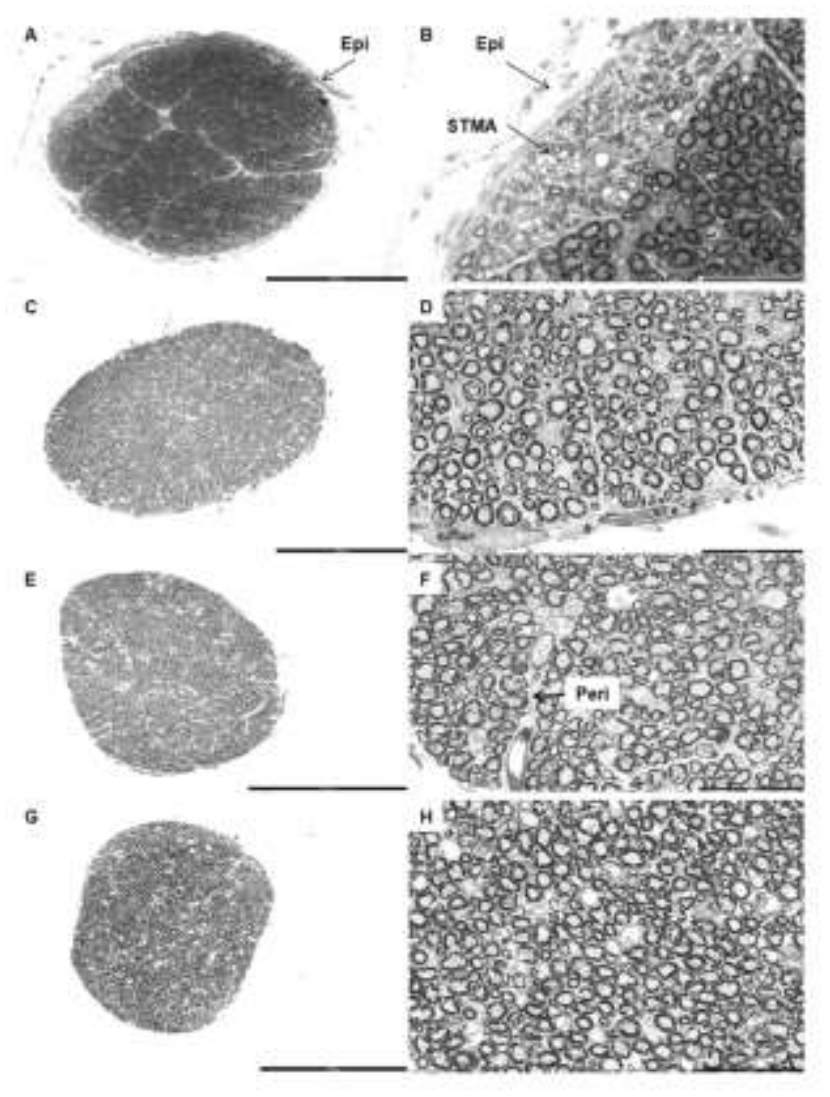

Figure 1. Cross-sections of the trochlear nerve in various age groups. Panel of low magnification (left column) and high magnification (right column) photomicrographs of toluidene blue stained semithin resin sections of trochlear nerves showing the Epineurium (Epi), Perineurium (Peri) and a bundle of Small Thinly Myelinated Axons (STMA). The darkly stained profiles seen in the micrographs are the myelin sheaths surrounding the axons of the trochlear nerve. A. and B. are of a 14 year old female; C. and D. are of a 38 year old female; E. and F. are of a 51 year old male and $\mathrm{G}$. and $\mathrm{H}$. are of a 71 year old male. Scale bar: $\mathrm{A}=\mathrm{C}=\mathrm{E}=\mathrm{G}=300 \mu \mathrm{m}$. $\mathrm{B}=\mathrm{D}=\mathrm{F}=\mathrm{H}=50 \mu \mathrm{m}$.

\section{Statistical Analysis}

We used mean (SD) to represent the data and analyzed them by using the statistical software SPSS (version 15.0, Chicago, Illinois). We compared the data with respect to different age groups using one-way analysis of variance (ANOVA) and post hoc comparison by using Bonferroni multiple comparison tests. A ' $p$ ' value of less than 0.05 was taken as significant. Unfortunately, we were unable to undertake two-way ANOVA to check for the effect of gender difference on the various parameters because groups $\mathrm{C}$ and $\mathrm{D}$ contained exclusively male subjects. 

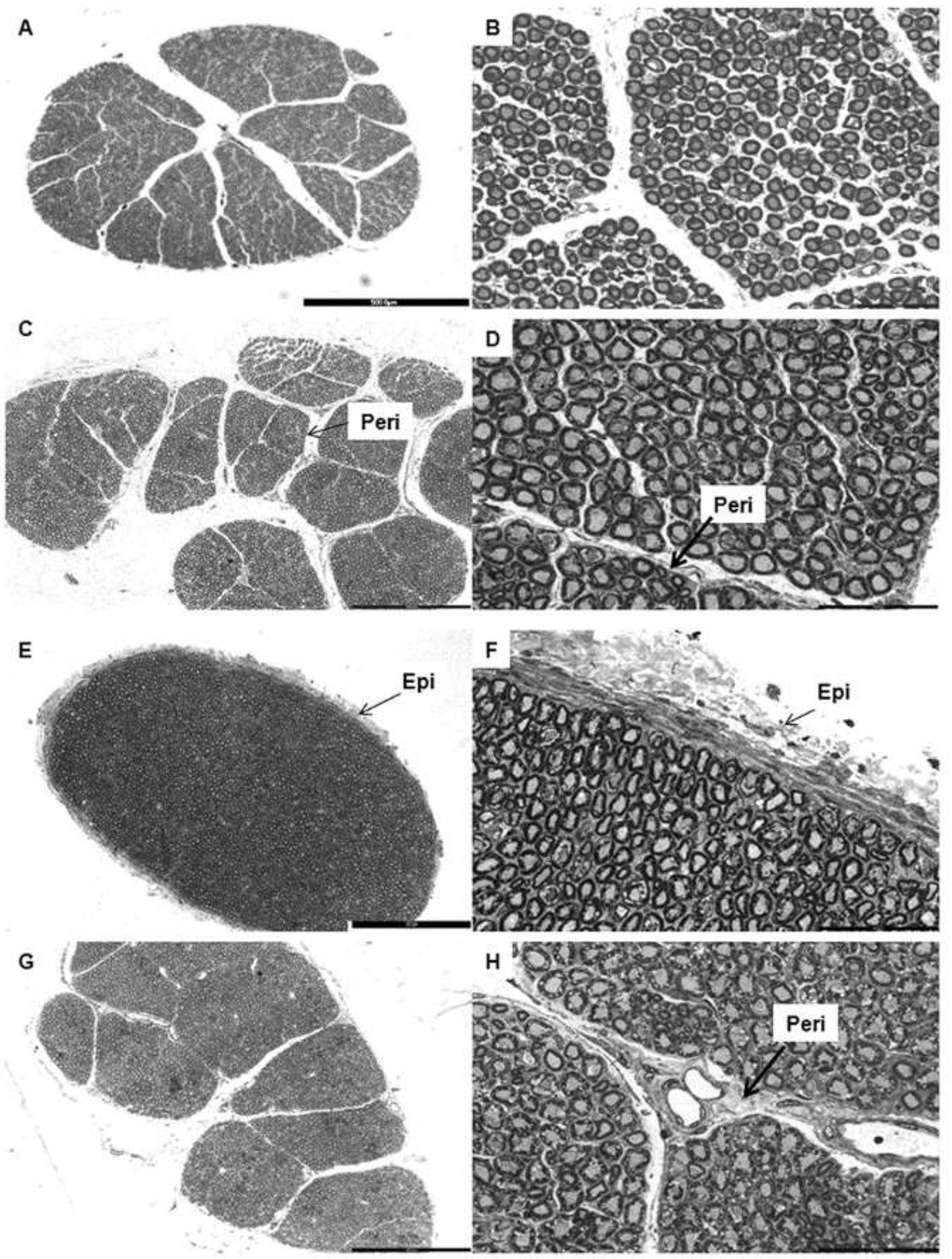

Figure 2. Cross-sections of the abducens nerve in various age groups. Panel of low magnification (left column) and high magnification (right column) photomicrographs of toluidene blue stained semithin resin sections of trochlear nerves showing the Epineurium (Epi) and Perineurium (Peri). The darkly stained profiles seen in the micrographs are the myelin sheaths surrounding the axons of the abducens nerve. A. and B. are of a 16 year old female; $\mathrm{C}$. and D. are of a 24 year old female; $\mathrm{E}$. and $\mathrm{F}$. are of a 45 year old male and $\mathrm{G}$. and $\mathrm{H}$. are of a 61 year old man. Scale bar: $\mathrm{A}=500 \mu \mathrm{m}$. $\mathrm{C}=\mathrm{E}=\mathrm{G}=$ $300 \mu \mathrm{m} . \mathrm{B}=\mathrm{D}=\mathrm{F}=\mathrm{H}=50 \mu \mathrm{m}$. 


\section{Table 1: Morphometric parameters of human trochlear nerve}

\begin{tabular}{|c|c|c|c|c|c|c|c|c|c|}
\hline \multirow{2}{*}{$\begin{array}{l}\text { Age } \\
\text { Groups } \\
\text { (Years) }\end{array}$} & \multirow{2}{*}{$\begin{array}{l}\text { No. of } \\
\text { samples }\end{array}$} & \multirow{2}{*}{$\begin{array}{l}\text { Mean cross } \\
\text { sectional area } \\
\text { of nerve } \\
\left(\mathrm{mm}^{2}\right)(5 \mathrm{D})\end{array}$} & \multirow{2}{*}{$\begin{array}{l}\text { Meanestimated } \\
\text { myelinated fibers } \\
\text { (SD) }\end{array}$} & \multirow{2}{*}{$\begin{array}{l}\text { Mean axonal } \\
\text { area of } \\
\text { myelinated } \\
\text { fibers ( } \mathrm{mm}^{3} \text { ) } \\
\text { (SD) }\end{array}$} & \multirow{2}{*}{$\begin{array}{c}\text { Axonal } \\
\text { Diameter } \\
\text { (pm) }\end{array}$} & \multirow{2}{*}{$\begin{array}{l}\text { Mean myelin } \\
\text { thickness } \\
\text { (pin) (SD) }\end{array}$} & \multicolumn{3}{|c|}{ Percentage distribution of fibers ( 6 ) } \\
\hline & & & & & & & Smail & Medium & Large \\
\hline $\begin{array}{c}A \\
(0-20) \\
\end{array}$ & 5 & $\begin{array}{c}0.26 \\
(0.05)\end{array}$ & $\begin{array}{c}2280 \\
(68337)\end{array}$ & $\begin{array}{l}22,69 \\
(7,24)\end{array}$ & 5.37 & $\begin{array}{r}1.84 \\
(0.88)\end{array}$ & 25.61 & 30.49 & 43.9 \\
\hline$\frac{B}{(21-40)}$ & 5 & $\begin{array}{l}0.27 \\
\{0.08\}\end{array}$ & $\begin{array}{c}1920 \\
(294.96)\end{array}$ & $\begin{array}{l}23.27 \\
(7.36)\end{array}$ & 5.44 & $\begin{array}{c}2.42 \\
(0.71)^{\pi}\end{array}$ & 13.68 & 27.37 & 58.95 \\
\hline $\begin{array}{c}\mathrm{C} \\
(41-60)\end{array}$ & 5 & $\begin{array}{c}0.28 \\
(0.08)\end{array}$ & $\begin{array}{c}2400 \\
(758.29)\end{array}$ & $\begin{array}{l}24.13 \\
(3.38)\end{array}$ & 5.54 & $\begin{array}{c}2.46 \\
(0.53)^{=}\end{array}$ & 12.19 & 26.83 & 60.98 \\
\hline $\begin{array}{c}D \\
(>60)\end{array}$ & 5 & $\begin{array}{l}0.23 \\
\{0.06\}\end{array}$ & $\begin{array}{c}2140 \\
(541.29)\end{array}$ & $\begin{array}{l}19.45 \\
(2.64)\end{array}$ & 4.98 & $\begin{array}{c}2.48 \\
(0.39)^{a}\end{array}$ & 17.95 & 35.9 & 46.15 \\
\hline
\end{tabular}

For ease of understanding, the axonal diameter has been computed from the mean axonal area. One way ANOVA followed by post-hoc Bonferroni multiple comparison test $\left({ }^{\#} \mathrm{p}<0.0001\right)$

\section{RESULTS}

\section{General organization of the TN and $A N$}

The cross-sections of the nerves revealed various shapes, the most common being oval for the TN and circular for the AN, although on many occasions they were irregular or even reniform due to their thin epineurium.

\section{Organization of connective tissue coverings and myelinated nerve fibers}

The TN and AN were covered by a thin layer of dense irregular connective tissue- the epineurium, which ensheathed the nerve fascicles and contained blood vessels that had smaller branches penetrating the perineurium. The endoneureal tube, made of delicate connective tissue, enclosed individual axons and contained numerous capillaries. Connective tissue septae from the epineurium divided many of the nerves into fascicles (Figure 1-2). Nerves with a thicker epineurium had fewer fascicles (Figure 2E). In the TN, only $30 \%$ of the nerves had well defined fascicles; whereas the AN had an average of eight fascicles (Figure 2). The myelinated fibers in the fascicles were more compactly arranged in earlier age groups than in later ones in both TN and AN.

\section{Distribution of small thinly myelinated fibers}

Although most of the fibers in $\mathrm{TN}$ and $\mathrm{AN}$ were intermingled with each other, a discrete group of small thinly myelinated fibers was found as a separate fascicle in the TN samples up to 40 years of age (Figure 1B). The loose connective tissue around these isolated fascicles appeared lighter stained. In the older age groups, this fascicle was no longer distinguishable as a separate entity.

In the AN, such a separate fascicle of small thinly myelinated fibers was not evident. However, the AN up to 40 years of age, had such fibers predominantly distributed towards the periphery of the nerve; whereas above 41 years similar fibers were found around the central region.

\section{Trochlear nerve-quantitative analysis}

Cross-sectional area. The mean cross-sectional area in groups A, B, C and D are presented in Table 1. There was no significant difference in the cross sectional area of the $\mathrm{TN}$ across the age groups $(\mathrm{p}=0.594)$.

Estimated myelinated fiber count. The number of myelinated nerve fibers in the TN ranged from 1100 to 3000. The estimated mean myelinated fiber count in groups A, B, C and D are given in Table 1. There was no significant difference in the estimated myelinated axonal counts between the various age groups $(\mathrm{p}=0.626)$.

Axonal area of myelinated fibers. The axonal area of myelinated fibers of the TN ranged from $16.09 \mu \mathrm{m}^{2}$ to $34.60 \mu \mathrm{m}^{2}$ (diameter 4.53 to $6.64 \mu \mathrm{m}$ ). The mean axonal area of myelinated fibers in groups A, B, C and D is specified in Table 1 and here too there was no significant 
difference observed between the various groups $(\mathrm{p}=0.693)$.

Overall, the TN had $18 \%$ small, $29.5 \%$ medium and $52.5 \%$ large myelinated fibers (Table 1 ). There was no significant difference in the percentage distribution of the various myelinated fiber types between the age groups $($ Pearson chi square $=7.9886)(p=0.239)$.
Myelin thickness. The myelin thickness of the TN ranged from $1.57 \mu \mathrm{m}$ to $2.60 \mu \mathrm{m}$. The average myelin thickness in groups A, B, C and D are given in Table 1. The myelin thickness of group A (up to 20 years) was significantly different in comparison to the other groups $(\mathrm{B}, \mathrm{C}$ and $\mathrm{D})$ $(\mathrm{p}<0.0001)$ (Table 1).

Table 2: Morphometric parameters of human abducens nerve

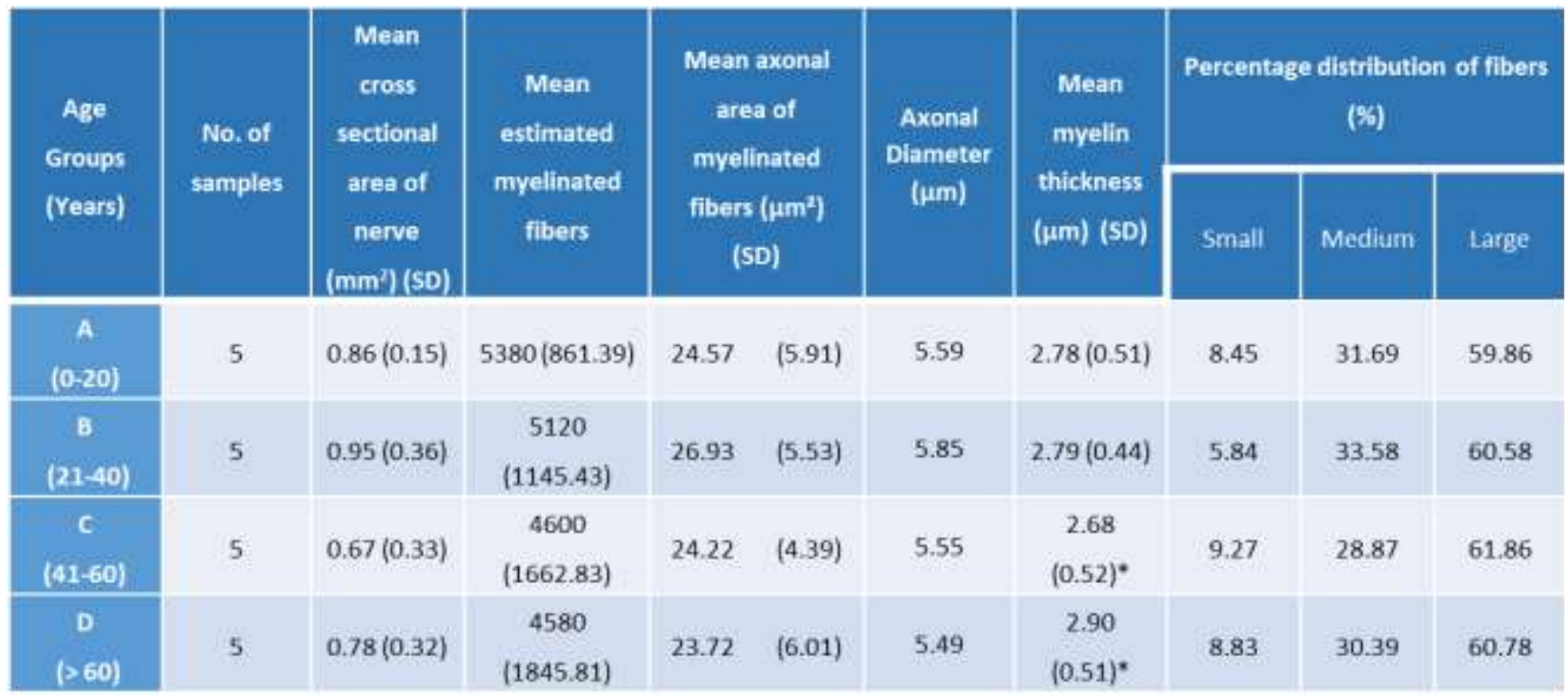

For ease of understanding, the axonal diameter has been computed from the mean axonal area. One way ANOVA followed by post-hoc Bonferroni multiple comparison test $(* \mathrm{p}<0.05)$

\section{Abducens nerve-quantitative analysis}

The AN appeared larger in cross-section, with many more nerve fascicles than the TN. But the epineurium of the AN was in general thinner than that of the TN.

Cross-sectional area The cross-sectional area of the AN varied from $0.36 \mathrm{~mm}^{2}$ to $1.32 \mathrm{~mm}^{2}$. The mean crosssectional area in groups A, B, C and D are mentioned in Table 2. There was no significant difference in the cross sectional area among the various age groups $(\mathrm{p}=0.457)$.

Estimated myelinated fiber count. The myelinated fiber count in the AN ranged from 1600 to 7000 . The estimated mean myelinated fiber count in groups A, B, C and D are presented in Table 2 and did not show any significant difference among the age groups $(\mathrm{p}=0.768)$.
Axonal area of myelinated fibers. The axonal area of the myelinated fibers of the AN varied from $14.35 \mu \mathrm{m}^{2}$ to $34.35 \mu \mathrm{m}^{2}$ (diameter 4.27 to $6.61 \mu \mathrm{m}$ ). The mean axonal area of the myelinated fibers in groups A, B, C and D are listed in Table 2. There was no significant difference in the mean axonal area of the myelinated fibers among the various age groups $(\mathrm{p}=0.799)$.

On the whole, the AN had $8 \%$ small, $31 \%$ medium and $61 \%$ large myelinated fibers (Table 2). There was no significant difference in the percentage distribution of different myelinated fibers across different age groups $($ Pearson chi square $=1.6060)(p=0.952)$.

Myelin thickness. The myelin thickness of the individual nerve fibers of AN ranged from $2.40 \mu \mathrm{m}$ to $3.36 \mu \mathrm{m}$. The mean myelin thickness in groups A, B, C and D are given in Table 2. Significant difference in myelin thickness was observed between groups C and D ( $\mathrm{p}=0.013$ ) (Table 2). 


\section{DISCUSSION}

This study provides morphometric data on the age related changes in the human TN and AN using state of the art modern stereological techniques. To the best of our knowledge this is the first report of the count of myelinated fibers using unbiased stereological methods in the human TN in the young, adult and old individuals.

\section{Methodology and Morphometry}

Most of the techniques used in earlier studies for estimating the morphological parameters of the nerves are biased to a variable extent. To overcome these methodological problems, stereological techniques such as the fractionator [25] have been successfully used to compute the number of myelinated fibers (Suppl. Fig. 1). Mayhew [26] and Larsen [19] further extended this application to get a precise estimate of parameters such as area, perimeter and myelin thickness of the nerve. In our laboratory, the developing human fetal cochlear nerve and age changes in the human oculomotor nerve have been studied successfully using unbiased stereology [7, 27]. This unbiased method represents an important advancement in quantitative morphological assessment of tissues and organs [28]. In the present study, fractionator and 2D nucleator were used to count and measure the myelinated nerve fibers without relying on any assumptions about their size, shape and orientation.

\section{Organization of connective tissue and nerve fascicles}

The connective tissue organization of the TN and AN is of great clinical importance as it provides the primary structural framework, biomechanical protection and also acts as a vehicle for carrying blood vessels to the nerve. The ill-defined fascicles noted in the cisternal portion of these nerves in all age groups may explain why this portion of these nerves is more prone to compressive forces. These fibers derive their blood supply from pial blood vessels that predispose them to ischaemia during stretching and compression [29]. The early onset of the sixth cranial nerve palsy seen in cases of raised intracranial pressure [30] could be explained by the thin and fragile epineurium of the $\mathrm{AN}$, as noted in the present study. The scanty connective tissue support, noted in the present study, in these nerves could predispose them to easier disruption of the blood nerve barrier [31-32].

\section{Distribution of small thinly myelinated fibers}

We found a characteristically identifiable bundle of small thinly myelinated fibers in the TN samples up to 40 years of age. Even though small thinly myelinated fibers were identifiable in the older age groups, however, they were integrated in the main nerve mass. A similar group of putative pupillomotor fibers was noted in the third cranial nerves [7, 33-34]. Similar group of fibers, of undetermined origin were observed in fetal trochlear nerves [35] and it was speculated that these fibers could be either the nervi tentori- a meningeal branch of the ophthalmic nerve, or sympathetic fibers from the internal carotid plexus. It may be argued that these could be artifacts of processing; however, the consistent appearance of these bundles in the younger age group proves that this is more than an artifact. In order to determine the nature of these distinct group of fibers, both in the $\mathrm{ON}$ and $\mathrm{TN}$, further studies involving a greater number of specimens, and exhaustive sectioning and examination of the entire cisternal segment of these cranial nerves is needed. Immunohistochemical and lesion studies in animal models will confirm our observations.

In the AN, such a separate fascicle was not visible, but we did notice that there was a difference in the distribution of small thinly myelinated nerve fibers across the ages. In the younger age groups these fibers were located more peripherally, whereas in the older age groups they were located more centrally. As observed in the ON, it may be likely that there is a redistribution of small thinly myelinated fibers in the AN with age or anatomical location [34]. This requires confirmation by exhaustive serial sectioning and immunohistochemical study for different fiber types (cholinergic/catecholaminergic) in the entire cisternal segment of the AN.

\section{Counts and measurements}

\section{Cross sectional area}

There are very few morphometric studies available related to the adult human TN and AN [33, 35]. A recent radiologic study reported that the average diameter of the adult TN and AN ranged from 0.75 to $1 \mathrm{~mm}$ and 0.43 to $1.85 \mathrm{~mm}$, respectively [13] which amounts to an area of 0.44 to $0.78 \mathrm{~mm}^{2}$ and 0.14 to $2.68 \mathrm{~mm}^{2}$ for the $\mathrm{TN}$ and AN. Radiologic studies are usually conducted on live persons and the plane of the images are parallel to a given reference plane. Therefore, if the nerve has a sinuous course, it is likely that the diameter of the nerve may be over-estimated due to sections being oblique to the longitudinal axis of the nerve whereas, in our study the cisternal portion of the TN and AN has been positioned in such a way that the cutting surface would be always perpendicular to the longitudinal axis of the nerve. The discrepancy between the available radiological data and histological data of the present study (Tables 1-2) may also be due to shrinkage of the tissue during histological 
processing, even though the tissue shrinkage is least with resin embedding [36-38].

\section{Trochlear nerve}

\section{Myelinated fibers count}

Ocular motor nerves contain efferent and afferent fibers that may be either myelinated or unmyelinated. Reports in literature indicate that myelinated fibers predominate in these nerves [7, 39]. Our findings (Table $1 \& 2$ ) are not quite in agreement with previous studies $[33,35]$ that were on electron microscopic montages, and counting frames were chosen manually, therefore were inherently biased. The unbiased stereological fractionator probe used in the present study enabled us to count the myelinated fibers without relying on any assumptions about their size, shape or orientation $[19,40-41]$; therefore, the results of the present study are more reliable.

\section{Mean axonal area of myelinated fibers, fiber composition and myelin thickness of TN}

In the present study, axonal area of myelinated fibers and myelin thickness was calculated using 2D nucleator probe, which showed no significant difference in the axonal area of myelinated fibers between the various age groups (Tables 1-2). Therefore, it can be concluded that there is very little change in the fiber morphology with aging. Ocular motor myelinated nerve fibers were classified according to their axonal area into small $(<10$ $\left.\mu \mathrm{m}^{2}\right)$, medium $\left(10-20 \mu \mathrm{m}^{2}\right)$ and large fibers $\left(>20 \mu \mathrm{m}^{2}\right)$ [7]. In our study, we found that the $\mathrm{TN}$ was predominantly made of large fibers as also seen by Sunderland and Hughes [33].

There was a significant difference in the myelin thickness between the younger and all other older age groups (Table 1). Myelin thickness of the human TN apparently increases with age, probably due to addition of newer lamellae [10]. However, in our light microscopic study we observed that in the older age groups (Groups B, $\mathrm{C}$ and $\mathrm{D}$ ) there was no significant change in the myelin thickness with increasing age. This finding needs to be confirmed by morphometry on transmission electron microscopic pictures using the 2D nucleator probe. The only available data for comparison of myelin thickness in the human TN was in samples of a fetus (2.8 months before birth) where it was $1.00 \mu \mathrm{m}$ and in a 17 year old, in which it was $2.00 \mu \mathrm{m}$. However, these were manually measured using an ocular micrometer [42].

\section{Abducens Nerve}

\section{Myelinated fibers count}

The present study is the first precise report on the count of myelinated fibers in the human AN covering a wide range of age groups (Table 2). The counts in the present study are higher than that reported by Sawabe et al. [39] in aged (63-88 years old) subjects, in which the counts ranged from 1473 to 2638 . This discrepancy in the findings may be due to the methodological disparity in the two studies, the latter having been done using older morphometric methods using camera lucida drawings and customised programs [39].

\section{Mean axonal area of myelinated fibers, myelin thickness and fiber composition of $A N$}

In the present study there was no significant change in the axonal area of myelinated fibers with age in AN (Table 2 ), but there was a significant increase in the myelin thickness of AN between group C and group D (Table 2) $(\mathrm{p}<0.05)$. But to come to a definite conclusion regarding an increased myelin thickness, further studies with a greater number of samples and electron microscopy needs to be undertaken [43].

Since the groups were not uniform in terms of male and female subjects, we cannot conclude that the significant differences observed in myelin thickness both in the TN and AN were due solely to differences in age.

\section{CONCLUSION}

In our study, the ocular motor nerves do not show evident microscopic changes due to aging except with respect to myelin thickness. Reason for this apparent robustness of the ocular nerves a propos aging may be due to the high level of motor activity in the muscles supplied by these nerves as suggested by Sturrock [44]. There are differing schools of thought about the relationship between gaze disorders and aging [45-50]; however, after the studies in our laboratory on all three ocular motor nerves (III, IV and VI), we believe that there may be other factors that affect the function of these nerves that eventually cause diplopia and different gaze disorders [7].

\section{ACKNOWLEDGEMENTS}

We acknowledge the contribution of the technical staff of the department of Forensic Medicine, and Electron Microscopic Facility of the Department of Anatomy at All India Institute of Medical Sciences, New Delhi, India. The basic infrastructure of the department of Anatomy was used for this study. 


\section{REFERENCES}

[1] Glimcher PW (2003). Eye movements. In:Squire LR, Bloom FE, McConnel SK, Roberts JL, Spitzer NC, Zigmond MJ, editors. Fundamental Neuroscience, 2nd Edn. New York: Academic Press. pp. 873-892.

[2] Calisaneller T, Ozdemir O, Altinors N (2006). Posttraumatic acute bilateral abducens nerve palsy in a child. Childs Nerv Syst, 22: 726-728.

[3] Dwarakanath S, Gopal S, Venkataramana NK (2006). Post-traumatic bilateral abducens nerve palsy. Neurol India, 54: 221-222.

[4] Kurbanyan K, Lessell S (2008). Intracranial hypotension and abducens palsy following upper spinal manipulation. Br J Ophthalmol, 92: 153-155.

[5] Hanu-Cernat LM, Hall T (2009). Late onset of abducens palsy after Le Fort I maxillary osteotomy. Br J Oral Maxillofac Surg, 47: 414-416.

[6] Brazis PW (1993). Palsies of the trochlear nerve: diagnosis and localization- recent concepts. Mayo Clin Proc, 68: 501-509.

[7] Sharma S, Ray B, Bhardwaj D, Dwivedi AK, Roy TS (2009). Age changes in the human oculomotor nerve. A stereological study. Ann Anat, 191: 260-266.

[8] Jacobson DM, Moster ML, Eggenberger ER, Galetta SL, Liu GT (1999). Isolated trochlear nerve palsy in patients with multiple sclerosis. Neurology, 53: 877879.

[9] Barr D, Kupersmith MJ, Turbin R, Bose S, Roth R (2000). Isolated sixth nerve palsy: an uncommon presenting sign of multiple sclerosis. J Neurol, 247: 701-704.

[10] Peters GB 3rd, Bakri SJ, Krohel GB. (2002). Cause and Prognosis of nontraumatic sixth nerve palsies in young adults. Ophthalmology, 109: 1925-1928.

[11] Keane JR (1993). Fourth nerve palsy: historical review and study of 215 inpatients. Neurology, 43: 24392443.

[12] Anwar S, Nalla S, Fernando DJ (2008). Abducens nerve palsy as a complication of lumbar puncture. Eur J Intern Med, 19: 636-637.

[13] Adams ME, Linn J, Yousry I (2008). Pathology of the Ocular Motor Nerves III, IV, and VI. Neuroimag Clin N Am, 18: 261-282.

[14] Jefferson G (1947). Isolated oculomotor palsy caused by intracranial aneurysm. Proc R Soc Med, 40: 419432.

[15] Rucker CW (1966). The causes of paralysis of the third, fourth and sixth cranial nerves. Am J Ophthalmol, 61: 1293-1298.

[16] Burger LJ, Kalvin NH, Smith JL (1970). Acquired lesions of the fourth cranial nerve. Brain, 93: 567-574.

[17] Ferreira T, Verbist B, van Buchem M, van Osch T, Webb A (2010). Imaging the ocular motor nerves. Euro J Radiol, 74: 314-322.

[18] Sterio DC (1984). The unbiased estimation of number and sizes of arbitrary particles using the disector. $\mathrm{J}$ Microsc, 134: 127-136.
[19] Larsen JO (1998). Stereology of nerve cross sections. J Neurosci Meth, 85: 107-118.

[20] Howard CV, Reed MG (1998). Random sampling and random geometry. In: Unbiased stereology- three dimensional measurement microscopy. SpringerVerlag: New York, NY. pp. 19-33.

[21] Mayhew TM, Sharma AK (1984). Sampling schemes for estimating nerve fiber size. I. Methods for nerve trunks of mixed fascicularity. J Anat, 139: 45-58.

[22] Mayhew TM, Sharma AK (1984). Sampling schemes for estimating nerve fiber size. II. Methods for unifascicular nerve trunks. J Anat, 139: 59-66.

[23] Gundersen HJG (1977). Notes on estimation of numerical density of arbitrary profiles: the edge effect. J Microsc, 111: 219-223.

[24] Gundersen HJG (1988). The nucleator. J Microsc 15: 3-21.

[25] Gundersen HJG (1986). Stereology of arbitrary particles. A review of unbiased number and size estimators and the presentation of some new ones, in memory of William R. Thompson. J Microsc, 143: 345.

[26] Mayhew TM (1988). An efficient sampling scheme for estimating fiber number from nerve cross sections: The fractionators. J Anat, 157: 127-134.

[27] Ray B, Roy TS, Wadhwa S, Roy KK (2005) Development of the human fetal cochlear nerve: a morphometric study. Hear Res, 202: 74-86.

[28] Schmitz C, Hof PR (2005). Design-based stereology in neuroscience. Neuroscience, 130: 813-831.

[29] Miller NR (2005). Walsh and Hoyts Clinical NeuroOphthalmology Vol 1. Williams \& Wilkins: Baltimore, MD. pp. 969-1040.

[30] Caramel JP, Bonnel F, Rabischong P (1983). The oculomotor nerves: biometry and endoneural fascicular systematization. Anat Clin, 5: 159-168.

[31] Thomas PK (1963). The connective tissue of peripheral nerve: an electron microscope study. J Anat, 97: 35-44.

[32] Allt G, Lawrenson JG (2000). The blood-nerve barrier: enzymes, transporters and receptors--a comparison with the blood-brain barrier. Brain Res Bull, 52: 1-12.

[33] Sunderland S, Hughs ESR (1946). The pupilloconstrictor pathway and the nerves to the ocular muscles in man. Brain, 69: 301-309.

[34] Kerr FWL, Hollowell OW (1964). Location of pupillomotor and accommodation fibers in the oculomotor nerves: experimental observations of paralytic mydriasis. J Neurol Neurosurg Psychiatry, 27: 473-481.

[35] Mustafa GY, Gamble HJ (1979). Changes in axonal numbers in developing human trochlear nerve. J Anat, 128: 323-330.

[36] Schuz A, Palm G (1989). Density of neurons and synapses in the cerebral cortex of the mouse. J Comp Neurol, 286: 442-445.

[37] Miettinen RA, Kalesnykas G, Koivisto EH (2002). Estimation of the total number of cholinergic neurons 
containing estrogen receptor- in the rat basal forebrain. J Histochem Cytochem, 50: 891-902.

[38] Edwards K, Griffiths, D, Morgan J, Pitman R, von Ruhland C (2009). Can the choice of intermediate solvent or resin affect glomerular basement membrane thickness? Nephrol Dial Transplant, 24: 400-403.

[39] Sawabe Y, Matsumoto K, Goto N, Otsuka N, Kobayashi N (1998). Morphometric nerve fiber analysis and aging process of the human abducent nerve. Okajimas Folia Anat Jpn, 74: 337-343.

[40] Geuna S (2000). Appreciating the difference between design-based and model-based sampling strategies in quantitative morphology of the nervous system. J Comp Neurol, 427: 333-339.

[41] Kaplan S, Geuna S. Ronchi G, Ulkay MB, von Bartheld CS (2010). Calibration of the stereological estimation of the number of myelinated axons in the rat sciatic nerve: A multicenter study. J Neurosci Methods, 187: 90-99.

[42] Schroder JM, Bohl J, Von Bardeleben U (1988). Changes of the ratio between myelin thickness and axon diameter in human developing sural, femoral, ulnar, facial and trochlear nerves. Acta Neuropathol, 76: 471-483.

[43] Peters A (2002). The effects of normal aging on myelin and nerve fibers: a review. J Neurocytol, 31 : 581-593.
[44] Sturrock RR (1989). Stability of neuron and glial number in the abducens nerve nucleus of the ageing mouse brain. J Anat, 166: 97-101.

[45] Huaman AG, Sharpe JA (1993). Vertical saccades in senescence. Invest Ophthalmol Vis Sci, 34: 25882595.

[46] Moschner C, Baloh RW (1994). Age-related changes in visual tracking. J Gerontol, 49, M235-238.

[47] Oguro H, Okada K, Suyama N, Yamashita K, Yamaguch S, Kobayashi S (2004). Decline of vertical gaze and convergence with aging. Gerontology, 50: $177-178$

[48] Qing Y, Kapoula Z (2004). Saccade-vergence dynamics and interaction in children and adults. Exp Brain Res, 156: 212-223.

[49] Rambold H, Neuman G, Sander T, Helmchen C (2005). Age-related changes of vergence under natural viewing conditions. Neurobiol Aging 27:163-172.

[50] Yang Q, Kapoula Z (2008). Aging does not affect the accuracy of vertical saccades nor the quality of their binocular coordination: a study of a special elderly group. Neurobiol Aging, 29: 622-638.

\section{Footnote}

Abbreviations used: AN- abducens nerve, ONoculomotor nerve, $\mathrm{TN}$ - trochlear nerve

\section{SUPPLEMENTARY}

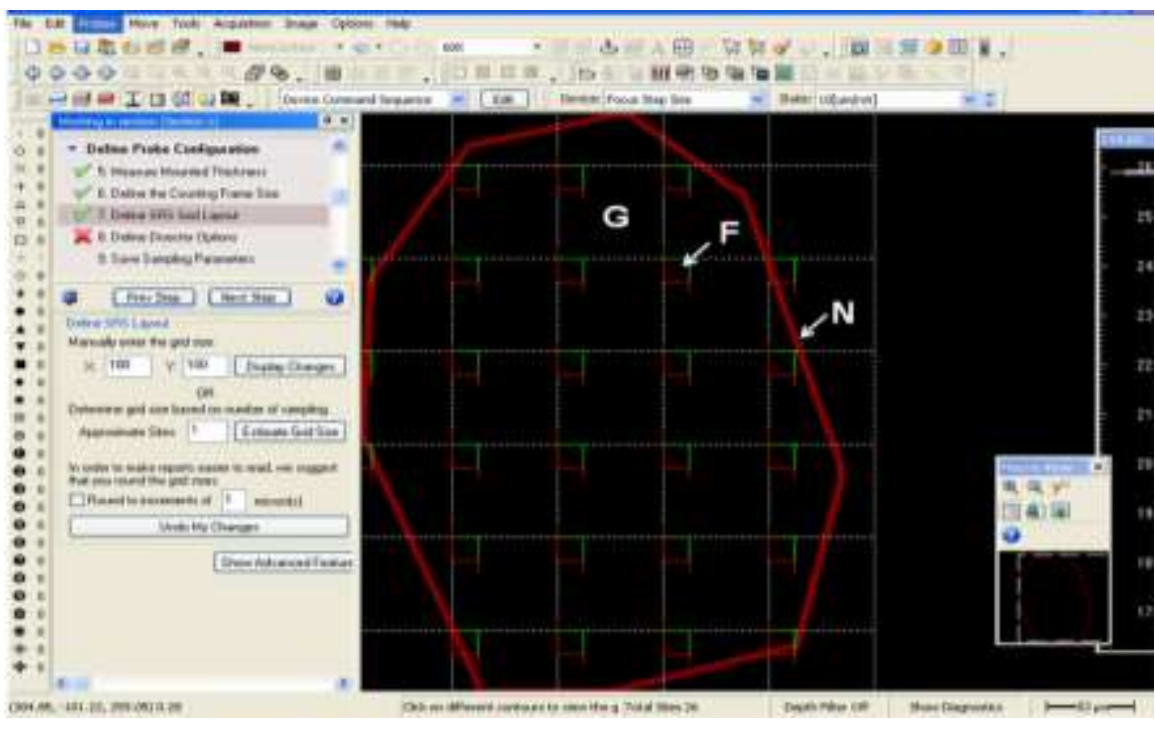

Supplementary Figure 1. Print screen of Optical fractionator and Nucleators in StereoInvestigator Software. This figure illustrates the optical fractionator and the nucleator methods. The unbiased counting frame is used with the fractionator probe. Axonal profiles contained either within the frame or touching the green lines were counted; whereas those that touched the 'forbidden' red lines were excluded. For the nucleator, a uniform random point within the profile of the axon is used to generate isotropic rays (in yellow) and the distances from the sampled point to the boundaries of the axon profile are marked (as depicted diagramtically in the left inset). 


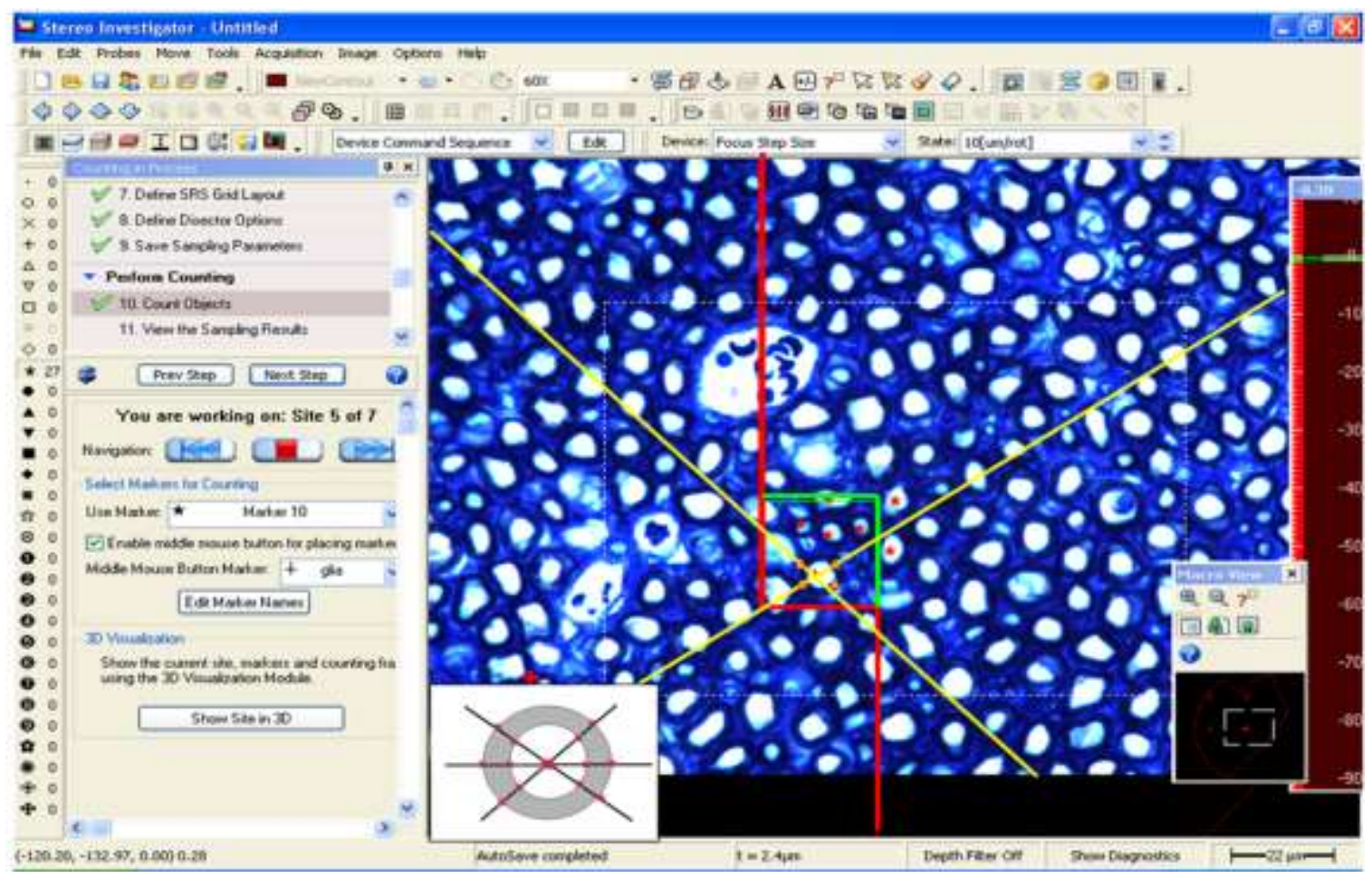

Supplementary Figure 2. Print Screen showing the morphometric program workflow in StereoInvestigator Software. This figure illustrates the nerve sectional profile contour $(\mathrm{N})$ showing the unbiased counting frame $(\mathrm{F})$ and the sampling grid (G). The contour was traced at a magnification of 100X using a BX61 motorized Olympus microscope. The ideal sampling grid size for this study was determined to be $250 \mu \mathrm{m}$ x $250 \mu \mathrm{m}$ and the unbiased counting frame was $25 \mu \mathrm{m} \times 25 \mu \mathrm{m}$. The axon profiles within the unbiased counting frame or touching its green lines were counted, whereas those that touched the 'forbidden' red lines were excluded. 